\title{
The Symbolic Computation of Differential Invariants of Polynomial Vector Field Systems Using Trees*
}

\author{
M. J. Doffou and R. L. Grossman ${ }^{\dagger}$ \\ Laboratory for Advanced Computing \\ University of Illinois at Chicago
}

\section{Abstract}

Let $K$ denote a field of characteristic 0 , and let $V=K^{N}$ denote the vector space over $K$ of dimension $N$. Let $R$ denote the $K$-algebra of polynomials over $V$;

$$
\begin{gathered}
r=\sum_{j_{1}=1}^{N} a_{j_{1}} x^{j_{1}}+\sum_{j_{1}, j_{2}=1}^{N} a_{j_{1}, j_{2}} x^{\jmath_{1}} x^{j_{2}}+\cdots, \\
a_{j_{1}}, a_{j_{1}, j_{2}}, \ldots \in K .
\end{gathered}
$$

Consider the algebra $\mathcal{D}$ of derivations of $R$. Given a derivation $E \in \mathcal{D}$, we are interested in symbolic algorithms for computing its invariants. To be more precise, the action of $G L(V)$ on $V$ induces an action on the space of coefficients $\mathcal{C}$ of the derivations. A polynomial over $\mathcal{C}$ is called invariant in case it is invariant under this action.

Our approach to computing differential invariants is to define an algebraic structure on the space of rooted, labeled trees $\mathcal{T}$ and introduce an algebra homomorphism from $\mathcal{C}$ to $\mathcal{T}$. Differential invariants are naturally expressed and easily computed in terms of a few basic operations on the space of trees.

Our main result is expressed in Theorem 5 and illustrated in Figure 2 and Table 2. It provides a simple and direct combinatorial means of computing differential invariants. The algorithm underlying Theorem 5 has been implemented in $\mathrm{C}++$.

We illustrate these ideas by computing all the differential invariants of vector field systems in the plane $V=K^{2}$. For example, for linear vector fields in the plane, there is a polynomial basis of invariants containing two polynomials, while for quadratic vector fields in the plane, there is a polynomial basis containing 16 polynomials [11].

\footnotetext{
${ }^{*}$ For further information, please contact Robert Grossman, Department of Mathematics, Statistics, and Computer Science, Mail Code 249. University of Illinois at Chicago, 851 South Morgan Street. Chicago, IL 60607, USA, 13124132176,13129961491 fax, grossman@uic.edu

${ }^{\top}$ This research was supported in part NASA grant NAG2-513 and NSF Grants DMS-8904740, IRI 9224605 and CDA 9303433. Part of this work was done while Robert Grossman was visiting the Department of Computer Science at Cornell University.

Permission to copy without fee all or part of this material is granted provided that the copies are not made or distributed for direct commercial advantages, the ACM copyright notice and the title of the publication and its date appear, and notice is given that copying is by permission of the Association for Computing Machinery. To copy otherwise, or to republish, requires a fee and/or specific permission. ISSAC'95 - 7/95 Montreal, Canada

(C) 1995 ACM 0-89791-699-9/95/0007 $\$ 3.50$
}

\section{Introduction}

Let $K$ denote a field of characteristic 0 , and let $V=K^{N}$ denote the vector space over $K$ of dimension $N$. Let $R$ denote the $K$-algebra of polynomials over $V$ :

$$
\begin{gathered}
r=\sum_{j_{1}=1}^{N} a_{j_{1}} x^{j_{1}}+\sum_{j_{1}, j_{2}=1}^{N} a_{j_{1}, j_{2}} x^{j_{1}} x^{j_{2}}+\cdots, \\
a_{j_{1}}, a_{j_{1}, j_{2}}, \ldots \in K .
\end{gathered}
$$

We are interested in the symbolic computation of invariants associated with derivations of $R$. By a derivation, we mean a linear map $E: R \longrightarrow R$ satisfying

$$
E(f g)=f E(g)+g E(f), \quad f, g \in R .
$$

Recall that a derivation $E$ of the polynomial algebra $R$ can be viewed as a vector field on the vector space $V$ with polynomial coefficients and is associated with the flows of the nonlinear system

$$
\dot{x}(t)=E(x(t)), \quad x(0)=x^{0} \in V .
$$

The hope is that algebraic invariants of $E$ provide information about the analytical properties of the flows of the system.

Note that a derivation of $E$ of $R$ has the form

$$
E=\sum_{\mu=1}^{N} r^{\mu}\left(x^{1}, \ldots, x^{N}\right) \frac{\partial}{\partial x^{\mu}}, \quad r^{\mu} \in R,
$$

and each polynomial $r^{\mu}$ is of the form given by Equation 1. Let $\mathcal{D}_{d, N}$ denote the vector space consisting of all derivations of $R$ such that each polynomial $r^{\mu}$ is of degree less than or equal to $d$.

To define invariants, we must work with the action on the coefficients of $r^{\mu}$ induced by an action on $V$. To do this, we need to define the coefficient space

$$
\mathcal{C}=\mathcal{C}_{d, N}=K^{M}, \quad M=N\left(1+N+N^{2}+\cdots+N^{d}\right),
$$

and a map

$$
\mathcal{D}_{d, N} \longrightarrow \mathcal{C}_{d, N}
$$

which sends a derivation $E$ to the point in $\mathcal{C}$ determined by its coefficients:

$$
E \mapsto a=\left(a_{j_{1}}^{\mu}, a_{j_{1}, j_{2}}^{\mu}, \ldots, a_{j_{1}, j_{2}, \ldots, j_{d}}^{\mu}\right) \in K^{M} .
$$




\begin{tabular}{|lll|}
\hline Space & Description & Typical Element \\
\hline $\mathcal{I}$ & invariants of $R$ defined via induced action on $\mathcal{C}$ & $p=p(a)$ \\
$\mathcal{P}$ & polynomials in $\mathcal{C}$ and $x$ 's & $\mathrm{p}=\mathrm{p}(\mathrm{a} ; \mathrm{x})$ \\
$\mathcal{C}$ & coefficients of derivations in $\mathcal{D}$ & $a=\left(a_{i_{1}}^{\mu}, \ldots\right)$ \\
$\mathcal{D}$ & derivations over $R$ & $E=E_{a}$ \\
$R$ & polynomials over $V$ & $r=\sum a_{i_{1}} x^{i_{1}}+$ \\
$V$ & points $v$ & $v=\sum \alpha_{\mu} e_{\mu}$ \\
\hline
\end{tabular}

Table 1: An action of $Q \in G L(V)$ induces an action on the coefficients $a \in \mathcal{C}$ of a derivation $E \in R$. Invariants are polynomials in the coefficients $a \in \mathcal{C}$ stable under this induced action. Note that each line in the table is defined using data from the line below.

Here all indices range from 1 to $N$ and the lower ones are symmetric.

Since we have introduced several spaces, we summarize some of these definitions in Table 1.

Under a linear change of variables in $V$,

$$
x=Q y, \quad Q \in G L(V)
$$

the corresponding nonlinear system

$$
\dot{x}(t)=E_{a}(x(t)), \quad \text { coeffs } a_{j_{1}, \ldots, j_{k}}^{i} \in \mathcal{C}
$$

is transformed into the nonlinear system

$$
\dot{y}(t)=E_{b}(y(t)), \quad \text { coeffs } b_{j_{1}, \ldots, j_{k}}^{i} \in \mathcal{C}
$$

We are interested in the

Problem A. Find polynomials $p$ over the vector space $\mathcal{C}=$ $\mathcal{C}_{d, N}$ such that

$$
p(a)=p(b), \quad \text { for all } Q \in G L(V),
$$

where $b \in \mathcal{C}$ is the vector of coefficients induced from $a \in \mathcal{C}$ by $Q \in G L(V)$. The polynomial $p$ is called a differential invariant of the nonlinear system 3 . Denote by $\mathcal{I}=\mathcal{I}_{d, N}$ the vector space over $K$ formed by all such polynomials.

We say that an invariant $p \in \mathcal{I}$ is reducible in case in can be written as a polynomial function of invariants of lower degree. A set of polynomials $\left\{p_{\alpha}\right\}$ is called a basis in case any invariant $p \in \mathcal{I}$ can be written as a polynomial in the $\left\{p_{\alpha}\right\}$, that is reducible with respect to the $\left\{p_{\alpha}\right\}$.

Problem B. Find a polynomial basis for $\mathcal{I}=\mathcal{I}_{d, N}$.

We emphasize that we are interested in algorithms useful for the explicit symbolic computation of differential invariants for specific systems. One knows from general principles [8] and [11] that differential invariants arise by viewing the coefficients of the vector fields as belonging to the appropriate tensor space and forming all contractions and alternations.

Our approach to computing differential invariants is to define an algebraic structure on the space of rooted, labeled trees $\mathcal{T}$ and introduce an algebra homomorphism from $\mathcal{C}$ to $\mathcal{T}$. Differential invariants are naturally expressed and easily computed in terms of a few basic operations on the space of trees.

Our main result is expressed in Theorem 5 and illustrated in Figure 2 and Table 2. It provides a simple and direct combinatorial means of computing differential invariants. The algorithm underlying Theorem 5 has been implemented and extended as part of the dissertation [2].
We illustrate these ideas by computing all the differential invariants of vector field systems in the plane $V=K^{2}$. For example, for linear vector fields in the plane, there is a polynomial basis of invariants containing two polynomials, while for quadratic vector fields in the plane, there is a polynomial basis containing 16 polynomials [11].

Our contribution in this paper is to point out that working with trees gives a simple, direct means for symbolically computing differential invariants for vector field systems. At this time, we can use trees to write down invariants (Problem A), but we do not have a simple means of identifying a basis (Problem B). Our method applies to polynomials coefficients in $\mathcal{C}_{d, N}$, for any degree $d$ and dimension $N$. We do not know of other explicit symbolic results for $d>2$ or $N>2$.

\section{Background and Related Work}

The theory of algebraic invariants, that is the theory of invariants of polynomials in $R$, has been well studied. Recently, there has been renewed interest, especially from the algorithmic viewpoint. Sturmfels in particular has developed a number of interesting relationships between algebraic invariant theory and the theory of Grobner bases [13].

The theory of differential invariants, that is the theory of invariants of differential polynomials in $\mathcal{D}$, is much less well understood. Closest to the viewpoint here, is the work of a school headed by Sibirsky and summarized in his book [11]. There the methods of alternation and contraction from classical tensor analysis are used to derive invariants and identities involving determinants are used to compute bases. In particular, the following theorem from [11] (expressed in the notation above) is closely related to our results:

Theorem 1 [7] and [11]. Polynomials $p \in \mathcal{P}$ obtained by applying alternation and total contraction to products of elements of $\mathcal{C}$ form a polynomial basis for invariants with respect to $G L(V)$.

We emphasize again that the general form of invariants is known from results such as this and the current challenge is to provide efficient algorithms for actually computing them.

Our approach here is to exploit the Hopf algebra structure of families of trees in order to organize the combinatorial computations required for the symbolic computation of differential invariants. The goal of using Hopf algebras as an organizing principle for combinatorial computations was articulated by Joni and Rota in [10]. The close connections between Hopf algebras and differential algebras was developed by Nichols and Weisfeiler in [9]. In a companion paper 
to the present one, we develop the differential algebra structure of families of trees [5], which underlies the approach here.

More specifically, it is known by the results of Gurevich [7], for the case of affine invariants $(Q \in G L(V))$, and by the results of Sibirsky and Taku [12], for the case of orthogonal invariants $(Q \in S O(V))$, that a basis of invariants is provided by alternating and contracting coefficients in $\mathcal{C}$.

\section{Algebras of Trees}

In this section, we review some material about trees and derivations from [1] and [4]. Although these results are not used explicitly below, several of the constructions below are more elaborate versions of this material.

Let $K$ denote the real or complex numbers. By a tree we will mean a finite rooted tree. Let $\mathcal{T}$ denote the $k$-vector space whose basis is the set of finite rooted trees. We now define an algebra structure on $\mathcal{T}$. Suppose that $\sigma_{1}, \sigma_{2} \in \mathcal{T}$ are trees. Let $\tau_{1}, \ldots, \tau_{r}$ be the children of the root of $\sigma_{1}$. If $\sigma_{2}$ has $n+1$ nodes (counting the root), there are $(n+1)^{r}$ ways to attach the $r$ subtrees of $\sigma_{1}$ which have $\tau_{1}, \ldots, \tau_{r}$ as roots to the tree $\sigma_{2}$ by making each $\tau_{i}$ the child of some node of $\sigma_{2}$. The product $\sigma_{1} \sigma_{2}$ is defined to be the sum of these $(n+1)^{r}$ trees. It can be shown that this product is associative, and that the trivial tree consisting only of the root is a right and left unit for this product.

It can also be shown that if each node of the tree (except for the root) is labeled with a derivation of $R$, then the same product turns the vector space whose basis consists of labeled trees into an algebra. For details, see [3]. We summarize this discussion with the following theorem.

Theorem 2 [3] (i) The vector space with basis the set of finite rooted trees is a graded algebra. (ii) The vector space with basis the set of finite rooted trees, all of whose nodes (except for the root) are labeled, is a graded algebra.

\section{P-Trees}

Recall that $R$ denotes the algebra of polynomials over $V$ :

$$
\begin{gathered}
r=\sum_{j_{1}=1}^{N} a_{j_{1}} x^{j_{1}}+\sum_{j_{1}, j_{2}=1}^{N} a_{j_{1}, j_{2}} x^{j_{1}} x^{j_{2}}+\cdots \\
a_{j_{1}}, a_{j_{1}, j_{2}}, \ldots \in K .
\end{gathered}
$$

A $P$-Tree over the polynomial algebra $R$ is a rooted, labeled tree satisfying

$\mathbf{P 1}$. The root has zero or more children; the children, if any, are labeled with the symbol $a$.

P2. The nodes which are labeled with the symbol $a$ may have zero or more children; the children, if any, are labeled with the symbol $x$.

P3. The nodes which are labeled with the symbol $x$ have no children.

We define the meld product of two P-Trees as the tree formed by identifying the roots of two P-Trees. It is immediate that the meld product is commutative and associative and that the unit consists of the P-Tree consisting of a single node. Let $\mathcal{T}(R)$ denote the vector space whose basis consists of $\mathrm{P}$-Trees over the polynomial algebra $R$. To summarize:
Theorem 3 The vector space $\mathcal{T}(R)$ whose basis consists of $P$-Trees over the polynomial algebra $R$ is a commutative $K$ algebra with respect to the meld product.

Recall that $\mathcal{C}$ denotes the coefficient space for derivations of $R$. Let $\mathcal{P}$ denote the polynomial algebra in the collections of indeterminates

$$
a \in \mathcal{C} \quad \text { and } \quad x^{1}, \ldots, x^{N} \in V^{*} .
$$

Recall that a differential invariant is simply an element of $\mathcal{P}$ which is invariant under the action on $\mathcal{P}$ induced by the action of $Q \in G L(V)$ on $V$.

We now define a linear map

$$
\psi: \mathcal{T}(R) \longrightarrow \mathcal{P}
$$

as follows:

1. Let $\alpha \in \mathcal{T}(R)$ be an element consisting of single tree. Let $e$ denote the number of children of the root.

2. Label each node of $\alpha$, except for the root, with an index label $\mu_{1}, \mu_{2}, \ldots$

3. Consider a child of the root labeled with the symbol $a$ and with the index label $\mu_{j}$. Assume that this node has children all labeled with symbol $x$ and with index labels $\mu_{i_{1}}, \ldots, \mu_{i_{k}}$. Define

$$
a\left(\mu_{j}\right)=a_{\mu_{i_{1}}, \ldots, \mu_{i_{k}}}^{\mu_{j}} x^{\mu_{i_{1}}} \cdots x^{\mu_{i_{k}}} .
$$

4. Define

$$
\psi(\alpha)=\sum a\left(\mu_{1}\right) \cdots a\left(\mu_{e}\right)
$$

where all indices are summed from $1, \ldots, N$

5. Extend $\psi$ by linearity to all of $\mathcal{T}(R)$.

It is immediate that:

Theorem 4 The map $\psi$ is a K-algebra homomorphism.

\section{D-Trees and Differential Invariants}

In this section, we introduce a simple construction on tensor products of $P$-trees which yields differential invariants. uct

Since $\mathcal{T}(R)$ is a $K$-algebra, we can form the tensor prod-

$$
\otimes \mathcal{T}(R)=\mathcal{T}(R) \otimes \cdots \otimes \mathcal{T}(R)
$$

Our construction for differential invariants is graph theoretic and depends upon connecting nodes of $\otimes \mathcal{T}(R)$ with dotted lines. In particular, we are interested in doing this in such a way that for all nodes, except for the roots, either D1 or D2 holds:

D1. A node labeled $x$ is connected with a dotted line to a node labeled $a$.

D2. Two nodes labeled $x$ are connected with dotted lines to a tree consisting of a single root.

Next, we need to define a linear map

$$
\rho: \bigotimes \mathcal{T}(R) \longrightarrow \mathcal{P}
$$

as follows: 
1. We first define $\rho$ for elements $\alpha$ of the domain which satisfy Properties D1 and D2 above.

2. Assume that $\alpha$ contains $k$ non-root nodes. Number each node with the index-label $\mu_{i}$, for $i=1,2, \ldots$, with the provision that if two nodes are connected with a dotted line then they share the same index-label.

3. If $\alpha$ contains a node labeled $a$, labeled with index label $\mu_{j}$ and has $j$ children with the index labels $\mu_{i_{1}}, \ldots, \mu_{i_{j}}$, then that node contributes

$$
\alpha(j)=a_{\mu_{i_{1}}, \ldots, \mu_{i_{j}}}^{\mu_{j}}
$$

to the sum below.

4. If $\alpha$ contains a tree with a single node which is attached with dotted lines to nodes labeled $\mu_{i_{1}}$ and $\mu_{i_{2}}$, then that node contributes

$$
\epsilon(j)=\epsilon^{\mu_{i_{1}}, \mu_{i_{2}}}
$$

to the sum below. Here $\epsilon$ is the alternating tensor on $V$ defined by $\epsilon^{\mu_{i_{1}}, \cdots, \mu_{i_{k}}}$ is equal to 1 , if $\left(\mu_{1}, \cdots, \mu_{k}\right)$ is an even permutation of $\{1, \ldots . N\}$; is equal to -1 , if it is an odd permutation; and is equal to 0 otherwise.

5. Form the sum

$$
\rho(\alpha)=\sum_{\mu_{1}, \ldots, \mu_{k}=1}^{N} \alpha(1) \cdots \alpha(k) \epsilon(1) \cdots \epsilon\left(k^{\prime}\right)
$$

6. For simplicity, in this paper, we do not define $\rho$ for elements which do not satisfy Properties D1 and D2.

For examples of this map see Figure 2 and the corresponding Table 2.

Theorem 5 Let $V=K^{N}$ and let $R$ denote the polynomial algebra over $R$. Consider elements $\alpha \in \bigotimes \mathcal{T}(R)$ connected with dotted lines satisfying Properties $D 1$ and $D 2$. Then $\rho(\alpha)$ is a differential invariant for a derivation of $R$ with respect to $G L(V)$.

Proof We only sketch the proof; for further details, see [6]. After unwinding notation, it follows immediately from Theorem 4 that dotted lines between a node labeled $a$ and a node labeled $x$ corresponds to contraction, while dotted lines between two nodes labeled $x$ corresponds to alternation. The fact that $\rho$ yields differential invariants then follows from Gurevich's Theorem (Theorem 1), proving the theorem.

\section{Experimental Results}

We developed a package in $\mathrm{C}++$ to compute differential invariants. The base package, TREE-ALG, implements a calculus for trees and is fairly complete. DIFF-INV is the package that actually compute invariants by enumerating tensor products $\alpha$ of labeled trees in $\otimes \mathcal{T}(R)$ which satisfy Properties D1 and D2 and computing the differential invariant $\rho(\alpha)$. The resulting differential invariants were then analyzed using standard symbolic systems such as Maple.

At the user level DIFF-INV provide a simple command line interface. The command inv $\mathrm{n} m \mathrm{k}$ computes the invariants of degree $k$ of an $n$-ary system of order $m$.

With Theorem 5 , it is straight forward to compute differential invariants for nonlinear systems in the plane $V=K^{2}$ elgreco\% inv 221

$\mathrm{n}:=2: \mathrm{d}:=2: \mathrm{k}:=1$ :

i $1:=\mathrm{a}(\mathrm{mo}, \mathrm{mo})$ :

elgreco\% inv 222

$\mathrm{n}:=2: \quad \mathrm{d}:=2: \quad \mathrm{k}:=2$ :

i1: $=\mathrm{a}(\mathrm{m} 1, \mathrm{mo}) * \mathrm{a}(\mathrm{mo}, \mathrm{m} 1)$ :

i $2:=a(m 0, m 0, m 1) * a\left(m 1, \mathrm{~m}_{1} 0_{0}, \mathrm{~m}_{1} 1\right) * e_{-}\left(\mathrm{m}_{1} 0_{-}, \mathrm{m}_{1} 1\right)$;

i $3:=a(m 0, m 1, m 0) * a\left(m 1, m 1 \_0, m 1 \_1\right) * e_{-}\left(m 1 \_0, m 1 \_1\right):$

$i 4:=\mathrm{a}\left(\mathrm{m} 0, \mathrm{~m} 0, \mathrm{~m} 0_{-} 1\right) * \mathrm{a}\left(\mathrm{m} 1, \mathrm{~m} 1, \mathrm{~m} 1_{-} 1\right) * \mathrm{e}_{-}\left(\mathrm{m} 0_{-} 1, \mathrm{~m} 11_{-} 1\right)$ :

i5: $=\mathrm{a}\left(\mathrm{m} 1, \mathrm{~m} 0, \mathrm{~m}_{-} 1\right) * \mathrm{a}\left(\mathrm{m} 0, \mathrm{~m} 1, \mathrm{~m}_{-} 1\right) * \mathrm{e}_{-}\left(\mathrm{m} 0_{-} 1, \mathrm{~m} 1{ }_{-} 1\right)$ :

$\mathrm{i} 6:=\mathrm{a}\left(\mathrm{m} 0, \mathrm{m0}, \mathrm{m} 0_{-} 1\right) * \mathrm{a}\left(\mathrm{m} 1, \mathrm{~m} 1 \_0, \mathrm{~m} 1\right) * \mathrm{e}_{-}\left(\mathrm{m} 0_{-} 1, \mathrm{~m} 1 \_0\right):$

$\mathrm{i} 7:=\mathrm{a}\left(\mathrm{m} 1, \mathrm{~m} 1{ }_{-} 0, \mathrm{mo}\right) * \mathrm{a}\left(\mathrm{mo}, \mathrm{m} 1, \mathrm{mo}_{-} 1\right) * \mathrm{e}_{-}\left(\mathrm{m} \mathrm{L}_{-} 1, \mathrm{~m} 11_{-} 0\right)$ :

is: $=\mathrm{a}\left(\mathrm{mo}, \mathrm{mo} 0_{-} 0, \mathrm{mo}\right) * \mathrm{a}\left(\mathrm{m} 1, \mathrm{~m} 1, \mathrm{~m} 1_{-} 1\right) * \mathrm{e}_{-}\left(\mathrm{m} 0_{-} \mathrm{o}_{\mathrm{m} 1} \mathrm{~m}\right)$ :

i $9:=\mathrm{a}\left(\mathrm{m} 1, \mathrm{~m} 0, \mathrm{~m} 1 \_1\right) * \mathrm{a}\left(\mathrm{m} 0, \mathrm{~m} 0_{-} 0, \mathrm{~m} 1\right) * e_{-}\left(\mathrm{m} 0_{-} 0, \mathrm{~m}_{-} 1\right):$

$\mathrm{i} 10:=\mathrm{a}\left(\mathrm{m0}, \mathrm{m} 0_{-} 0, \mathrm{m0}\right) * \mathrm{a}\left(\mathrm{m} 1, \mathrm{~m} 1_{-} \mathrm{O}, \mathrm{m} 1\right) * \mathrm{e}_{-}\left(\mathrm{mo} \mathrm{O}_{-} 0, \mathrm{~m} 1{ }_{-} 0\right):$

i11: $=\mathrm{a}\left(\mathrm{m} 1, \mathrm{~m} 1 \_0, \mathrm{~m} 0\right) * \mathrm{a}\left(\mathrm{mo}, \mathrm{m} 0{ }_{-} 0, \mathrm{~m} 1\right) * e_{-}\left(\mathrm{mo} 0_{-} 0, \mathrm{~m} 1 \_0\right):$

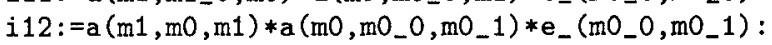

i1 $3:=\mathrm{a}(\mathrm{m} 1, \mathrm{~m} 1, \mathrm{m0}) * \mathrm{a}\left(\mathrm{m} 0, \mathrm{~m} 0_{-} 0, \mathrm{~m}_{-} 1\right) * \mathrm{e}_{-}\left(\mathrm{mo} 0_{-} 0, \mathrm{mO}_{-} 1\right)$ :

elgreco\% inv 231

$\mathrm{n}:=2: \mathrm{d}:=3: \mathrm{k}:=1:$

i1: $=\mathrm{a}(\mathrm{m} 0, \mathrm{mo}):$

i $2:=\mathrm{a}\left(\mathrm{m} 0, \mathrm{~m} 0, \mathrm{~m}_{-} 1, \mathrm{~m}_{-} \_2\right) * \mathrm{e}_{-}\left(\mathrm{m} 0_{-} 1, \mathrm{~m} 0_{-} 2\right)$ :

i3: $=\mathrm{a}\left(\mathrm{mo}, \mathrm{mo} \mathrm{O}_{0}, \mathrm{mo}, \mathrm{mo}_{-} 2\right) * \mathrm{e}_{-}\left(\mathrm{mo}_{-} \mathrm{O}, \mathrm{mo}_{-} 2\right)$ :

$i 4:=\mathrm{a}\left(\mathrm{m} 0, \mathrm{mO}_{-} \mathrm{O}, \mathrm{mO}_{-} 1, \mathrm{m0}\right) * \mathrm{e}_{-}\left(\mathrm{mO}_{-} \mathrm{O}, \mathrm{m} \mathrm{L}_{-} 1\right)$ :

Figure 1: The figure illustrates using DIFF-INV to compute the invariants of a nonlinear polynomial system.

whose coefficients are quadratic polynomials. This can be done either by hand or using DIFF-INV. See [11], for example and Table 2. Given the differential polynomials, one can then compute a basis using standard symbolic packages. It turns out that there are sixteen of them.

On the other hand, our package is quite helpful to write down similar invariants for cubic or higher coefficients or for $V=K^{N}$, where $N>2$. Unfortunately there are so many of them that standard symbolic packages cannot compute a basis, and our methods, at this time, are not powerful enough to compute a basis on the basis of properties of the trees. For results in this direction, see [2].

The program output use a linear notation $\mathrm{a}(\mathrm{k}, \mathrm{l}, \mathrm{n}, \ldots)$ to represent the tensor $a_{l n \ldots}^{k}$. The first index is the upper index, all others ones are lower indices. Similarly

$$
e_{-}(k, 1, n, \ldots)
$$

represents the alternation $\epsilon^{k l n \ldots}$. See Figure 1. 


$$
\begin{aligned}
I_{1} & =\sum a_{\mu_{1}}^{\mu_{1}} \\
I_{2} & =\sum a_{\mu_{2}}^{\mu_{1}} a_{\mu_{1}}^{\mu_{2}} \\
I_{3} & =\sum a_{\mu_{2}, \mu_{1}}^{\mu_{1}} a_{\mu_{3}, \mu_{4}}^{\mu_{2}} a_{\mu_{5}}^{\mu_{3}} \epsilon^{\mu_{3}, \mu_{5}} \\
I_{4} & =\sum a_{\mu_{2}, \mu_{1}}^{\mu_{1}} a_{\mu_{3}, \mu_{4}}^{\mu_{3}} a_{\mu_{5}}^{\mu_{2}} \epsilon^{\mu_{4}, \mu_{5}} \\
I_{5} & =\sum a_{\mu_{2}, \mu_{3}}^{\mu_{1}} a_{\mu_{1}, \mu_{4}}^{\mu_{3}} a_{\mu_{5}}^{\mu_{2}} \epsilon^{\mu_{4}, \mu_{5}} \\
I_{6} & =\sum a_{\mu_{2}, \mu_{3}}^{\mu_{1}} a_{\mu_{4}, \mu_{5}}^{\mu_{4}} a_{\mu_{6}}^{\mu_{2}} a_{\mu_{1}}^{\mu_{4}} \epsilon^{\mu_{2}, \mu_{6}}
\end{aligned}
$$

Table 2: The first six differential invariants for nonlinear systems in the plane $V=K^{2}$ with quadratic coefficients. All sums are from 1 to 2 .

$$
\text { द }
$$

11<smiles></smiles>

12
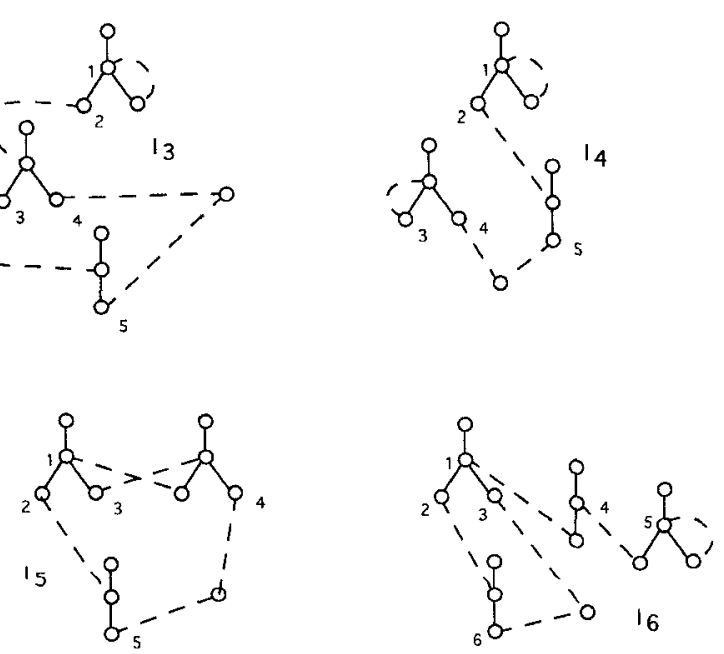

Figure 2: These are the trees corresponding to the first six differential invariants for planar systems with quadratic coefficients listed in Table 2. Except for $I_{2}$, this is the same invariant basis as in Sibirsky [11].

\section{Discussion and Conclusion}

The goal of this paper is to point out that a graph theoretic construction on labeled trees provides a simple and direct means of computing differential invariants for nonlinear differential equations with polynomial coefficients. We developed a package in $\mathrm{C}++$ implementing this algorithm and described the results for planar differential equations with quadratic coefficients.

For example, let $V=K^{2}$ denote the plane and let $R$ denote the space of polynomials over $V$ of degree 2 or less. There is a sixteen dimensional basis for $G L(V)$-invariants for derivations of $R$ [7]. This corresponds precisely to rooted, labeled trees in $\mathcal{T}(R)$ satisfying Properties D1 and D2. The latter are easy to compute.

Let $\mathcal{D}$ denote the $K$-algebra of derivations of $R$. The results here are closely related to the recent results in [5] which show that space of rooted trees, labeled with elements of $\mathcal{D}$ form what is called an $R / k$-bialgebra, which is the Hopf algebraic version of a differential algebra.

The results here are unsatisfying in that suitable properties of trees should lead directly to a basis of invariants. Work in this direction appears in [2].

\section{References}

[1] P.E. Crouch and R. L. Grossman, "The Explicit Computation of Integration Algorithms and First Integrals for Ordinary Differential Equations With Polynomial Coefficients Using Trees," Proceedings of the 1992 International Symposium on Symbolic and Algebraic Computation, ACM Press, pp. 89-94.

[2] M. J. Doffou, "Computing Differential Invariants Using Trees," Ph.D. Thesis, University of Illinois at Chicago, in preparation.

[3] R. Grossman and R. G. Larson, "Hopf-algebraic structure of families of trees," J. Algebra Volume 126, 1989, 184-210.

[4] R. L. Grossman and R. G. Larson, "The symbolic computation of derivations using labeled trees," Journal of Symbolic Computation, Volume 13, pp. 511-523, 1992.

[5] R. L. Grossman and R. G. Larson, " $R / k$-bialgebras of trees," submitted for publication.

[6] R. L. Grossman, "Differential Invariants and Trees," in preparation.

[7] G. B. Gurevich, Foundations of the Theory of Algebraic Invariants, GITTL, Mosow and Leningrad, 1948.

[8] D. Hilbert, Theory of Algebraic Invariants, Cambridge University Press, Cambridge, 1993.

[9] W. Nichols and B. Weisfeiler, Differential formal groups of J. F. Ritt, American Journal Mathematics, Volume 104, 1982, pp. 943-1003.

[10] S. A. Joni and G.-C. Rota, Coalgebras and bialgebras in combinatorics, Studies in Applied Mathematics, Volume 61, 1979, pp. 93-139; reprinted in "Umbral Calculus and Hopf Algebras," American Mathematical Society, Providence, 1982, pp. 1-47.

[11] K. S. Sibirsky, Introduction to the Algebraic Theory of Invariants of Differential Equations, Manchester University Press, Manchester, 1982. 
[12] K. S. Sibirsky and V. D. Taku, Algebraic Invariants of Many-Dimensional Differential Systems, DifferentisialNyje Uravnenija, Volume 12, pp. 281-291, 1988.

[13] B. Sturmfels, Algorithms in Invariant Theory, SpringerVerlag, Wien, 1993. 